\title{
Analysis of Chromosome Positions in the Interphase Nucleus of Chinese Hamster Cells by Laser-UV-Microirradiation Experiments
}

\author{
T. Cremer ${ }^{1}$, C. Cremer ${ }^{2}$, T. Schneider ${ }^{2}$, H. Baumann ${ }^{1}$, L. Hens ${ }^{3}$, and M. Kirsch-Volders ${ }^{3}$ \\ Institut für Anthropologie und Humangenetik, University of Heidelberg, Im Neuenheimer Feld 328, D-6900 Heidelberg, \\ Federal Republic of Germany \\ ${ }^{2}$ Institut für Humangenetik und Anthropologie, University of Freiburg i. Br., Albertstr. 11, D-7800 Freiburg, Federal Republic of Germany \\ ${ }^{3}$ Department of Human Genetics, Free University of Brussels (V.U.B.), Pleinlaan 2, B-1050 Brussels, Belgium
}

Summary. Unsynchronized cells of an essentially diploid strain of female Chinese hamster cells derived from lung tissue (CHL) were laser-UV-microirradiated $(\lambda=257 \mathrm{~nm})$ in the nucleus either at its central part or at its periphery. After $7-9 \mathrm{~h}$ postincubation with $0.5 \mathrm{mM}$ caffeine, chromosome preparations were made in situ. Twenty-one and 29 metaphase spreads, respectively, with partial chromosome shattering (PCS) obtained after microirradiation at these two nuclear sites, were Q-banded and analyzed in detail. A positive correlation was observed between the frequency of damage of chromosomes and both their DNA content and length at metaphase. No significant difference was observed between the frequencies of damage obtained for individual chromosomes at either site of microirradiation. The frequency of joint damage of homologous chromosomes was low as compared to nonhomologous ones. Considerable variation was noted in different cells in the combinations of jointly shattered chromosomes. Evidence which justifies an interpretation of these data in terms of an interphase arrangement of chromosome territories is discussed. Our data strongly argue against somatic pairing as a regular event, and suggest a considerable variability of chromosome positions in different nuclei. However, present data do not exclude the possibility of certain non-random chromosomal arrangements in CHL-nuclei. The interphase chromosome distribution revealed by these experiments is compared with centromere-centromere, centromere-center and angle analyses of metaphase spreads and the relationship between interphase and metaphase arrangements of chromosomes is discussed.

\section{Introduction}

Laser-microirradiation of the cell nucleus has been established as a new approach to investigation of the chromosome arrangement in interphase. Several procedures have been described to detect the microirradiated chromosome segments at the first postirradiation mitosis, and thus visualize the arrangement present at the site and time of microirradiation (Zorn et al. 1976, 1979; Cremer et al. 1980; Cremer et al. 1982). Data obtained by these methods have produced evidence for the idea that chromosomes occupy certain territories in the interphase nucleus of Chinese hamster cells, whereas the question of random or non-random arrangements of these territories awaits further clarification.

Offprint requests to: $\mathbf{T}$. Cremer
Here, we report on the evaluation of chromosomal damage observed after partial irradiation of the nucleus and caffeine post-treatment of essentially euploid Chinese hamster cells grown in vitro. By this treatment we have obtained fragmentation or pulverization of a few chromosomes in some cells, while in other cells shattering of the whole chromosome complement was obtained (Zorn et al. 1976). We refer to these two types of damage localization as partial (PCS) and generalized (GCS) chromosome shattering, respectively. Experimental conditions for the induction of PCS and GCS have been carefully studied (Cremer et al. 1981). Recently, we have demonstrated by indirect immunofluorescence microscopy that antibodies to UV-irradiated DNA react exclusively with the area of shattered chromatin in metaphases with PCS. In cells with GCS, significant fluorescence is also restricted to a small fraction of microirradiated chromatin, while the rest of the shattered chromosome complement remains unstained (Cremer et al. 1983). We have concluded that PCS and GCS indicate two levels of chromosome damage which can be produced by the synergistic action of ultraviolet light and caffeine. Level 1 as indicated by PCS appears to be restricted to the microirradiated part of the chromosome complement, while level 2 involves both microirradiated and non-irradiated chromosomes and results in GCS (Cremer et al. 1981 a, b, 1983).

On the basis of the evidence summarized above, we have used metaphases with PCS in the present investigation to elucidate the internal order of the interphase nucleus. Jointly damaged chromosomes in these metaphases are expected to reflect chromosomes situated closely together in the interphase nucleus at the site and time of microirradiation. The present investigation was particularly aimed at the question of whether (i) homologous association occurs in the interphase nucleus of Chinese hamster cells and (ii) chromosome territories of individual chromosomes occupy particular sites of the interphase nucleus. Somatic pairing has been shown to exist in cells of many plant species (Avivi and Feldmann 1980), but present evidence in mammalian species is conflicting (Comings 1980; Wollenberg et al. 1982). Our microbeam study was complemented by an analysis of the chromosome arrangement in metaphase spreads. Comparison of data obtained by both types of analysis gives an opportunity to compare the interphase and metaphase arrangement of chromosomes. Such a comparison is of considerable importance, since conclusions concerning the internal order of chromosomes in the interphase nucleus have been tentatively drawn in a large number of studies from the analysis of chromosome arrangements at metaphase (Hens et al. 1982; for review see Comings 1980). 


\section{Material and Methods}

Cell Strain and Culture Conditions. A fibroblastoid Chinese hamster cell (CHL) strain was established from lung tissue of a 3-week-old female and grown under standard conditions (Zorn et al. 1976). A number of early passage cultures were stored in liquid nitrogen and cells from passage numbers 10-15 were used for the experiments. At this passage level the majority of cells had still maintained their diploid status as shown by Q-banding analysis. The doubling time of exponentially growing cultures was approximately $30 \mathrm{~h}$. If not stated otherwise, experiments were performed with this cell strain.

Laser Microbeam and Conditions of Microirradiation. The UVlaser-microbeam $(\lambda=257 \mathrm{~nm})$ has been described in detail (Cremer et al. 1974, 1976). For microirradiation, 15-45 $\times 10^{3}$ cells were seeded in plastic petri dishes (Nunc, $\varnothing \cdot 5 \mathrm{~cm}$ ). In the middle of these dishes experimental fields of approximately $0.25 \mathrm{~mm}^{2}$ each were marked by scalpel cuts. Some $24 \mathrm{~h}$ later the dishes were placed in special irradiation chambers as previously described (Cremer et al. 1976, 1981) and cells in experimental fields were microirradiated in the nucleus, while cells outside these fields served as controls. The diameter of the microbeam was $1-2 \mu \mathrm{m}$ at its focal site. In each nucleus the nuclear area was microirradiated either at its central part or at its edge. The UV-power of the microbeam at the cell surface was approximately $7.5 \times 10^{-9} \mathrm{~W}$, the irradiation time was $1 / 1 \mathrm{~s} \mathrm{~s}$. Some 180-300 cells were microirradiated per petri dish at room temperature within a period of up to $1 \mathrm{~h}$. Some $10 \%$ of cells within experimental fields were excluded from microirradiation, mainly because the outline of the cell nucleus could not be clearly distinguished in phase contrast.

Post-Treatment of Microirradiated Cells. After microirradiation the cells received fresh medium with $0.5 \mathrm{mM}$ caffeine. In addition, ${ }^{3} \mathrm{H}$-thymidine $(0.05 \mu \mathrm{Ci} / \mathrm{ml}$, specific activity $5 \mathrm{Ci} /$ mmole, Amersham Buchler) was added in some experiments. Cells were postincubated at $37^{\circ} \mathrm{C}$ in a humidified atmosphere with $5 \% \mathrm{CO}_{2}$ for $7-9 \mathrm{~h}$. During the last $3 \mathrm{~h} 1 \mu \mathrm{g} / \mathrm{ml}$ colchicine was added to block cells in mitosis. Chromosome preparation of cells in situ was performed as previously described (Zorn et al. 1979). Air dried preparations were stained with quinacrine mustard (Caspersson et al. 1970). Banded metaphase spreads were photographed using a Zeiss photomicroscope equipped with epifluorescence illumination. For further evaluation cells were poststained with aceto-orcein. Autoradiography was performed as previously described (Zorn et al. 1979). As in previous publications of our group (Zorn et al. 1976, 1979; Cremer et al. 1982) the numbering system used for karyotyping of the Chinese hamster chromosomes is the one used by Kato and Yosida (1972), where the X chromosome is placed in sequence with the autosomes in descending size (compare Ray and Mohandas 1976).

Metaphase Chromosome Distribution Analysis. "Generalized distances" between the centromere of each chromosome and the center of the metaphase spread were calculated to determine the positions of metaphase chromosomes using the method of Barton and David (1962). The distribution of homologous chromosomes was evaluated in two ways. Generalized distances between the centromeres of homologous chromosomes were compared with the distances between non-homologous ones. In addition, an angle analysis was carried out by comparing angles established between the centromere of each chromosome, the center of the metaphase spread and the centromere of its

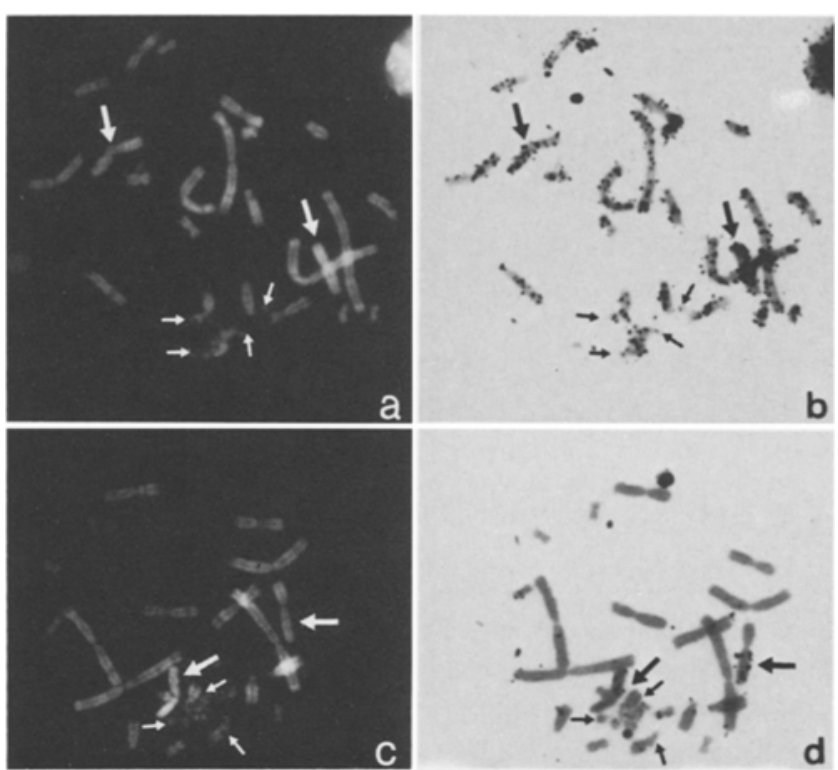

Fig. 1 a-d. Metaphase spreads with PCS were obtained after microirradiation of the nuclear edge and postincubation with caffeine $(0.5 \mathrm{mM})$ and ${ }^{3} \mathrm{H}$-thymidine $(0.05 \mu \mathrm{Ci} / \mathrm{ml})$ for $7 \mathrm{~h}$. Colchicine $(1 \mu \mathrm{g} / \mathrm{ml})$ was added after $4 \mathrm{~h}$. Note that ${ }^{3} \mathrm{H}$-thymidine was only added $1 \mathrm{~h}$ after the beginning of the experiment, when microirradiation of all cells in the experimental field of the petri dish had been finished. a, c Q-banded metaphase spreads stained with quinacrine-mustard. Small arrows indicate shattered chromosomes; large arrows point to the $\mathrm{X}$-chromosomes. $\mathbf{b}, \mathrm{d}$ The same metaphase spreads after autoradiography (exposure time 1 week) and poststaining with aceto-orcein. In $\mathbf{b}$ both shattered and intact chromosomes are labelled. In contrast, in d label is observed over the late replicating segments of the apparently intact X-chromosomes, but not over the shattered chromosome material

homologous counterpart or with any other non-homologous chromosome. Details of these methods, which include a circular transformation of centromere positions into an ideal circular image of the metaphase spread with unit radius, have been described extensively elsewhere (Hens 1976; Hens et al. 1982).

\section{Results}

Evaluation of Chromosome Damage in the First Postirradiation Mitosis after UV-Microirradiation of the Interphase Nucleus. Approximately 13,000 CHL-cells were microirradiated in nonsynchronized cultures either in the central area of the nucleus or at its edge. We refer to these two modes of microirradiation as "central" and "peripheral" microirradiation, respectively. The shape of the interphase nucleus of CHL-cells in vitro resembles a flat ellipsoid. In cases of peripheral microirradiation, chromatin constituting the nuclear edge where the curvature of the nuclear envelope is maximum was damaged, while in cases of central microirradiation chromatin constituting the central part of the nucleus was involved. It is important to note that in both cases some part of the nuclear envelope plus adjacent chromatin was hit by the microbeam. Accordingly, our experiments do not contribute to the question of which chromosomes or parts thereof are associated with the nuclear envelope and which are not. However, frequencies of shattering obtained for each chromosome of the complement after central or peripheral microirradiation should provide information about whether certain chromosomes are preferentially localized in the central area or at the nuclear edge. 
Table 1. Frequency of chromosome damage (classes A-E2) following central and peripheral microirradiation. Metaphase spreads obtained in experimental fields after central and peripheral microirradiation, respectively, and metaphase spreads from non-irradiated regions of the same petri dishes (control) were classified as previously described (Cremer et al. $1981 \mathrm{a}$ ). In class A, chromosomes appeared intact; in class $\mathrm{B}$, one or two chromosome aberrations were noted; in class $\mathrm{C}$, more than two aberrations were observed, but the majority of chromosomes appeared intact-this class comprises the cells with PCS (Figs. 1, 3); in class $\mathrm{D}$, shattering was observed in most chromosomes, but one or several chromosomes still appeared intact; in class $\mathrm{E}$, all chromosomes were shattered, appearing fragmented and/or pulverized (GCS); in subclass E1, fragments resembling parts of mitotic chromosomes could still be recognized at least in part of the spread; in subclass E2, the whole chromosome complement appeared pulverized

\begin{tabular}{llll}
\hline \multicolumn{4}{c}{ Site of microirradiation } \\
\cline { 2 - 4 } & $\begin{array}{l}\text { Central } \\
\left(n^{\mathrm{a}}=260\right) \\
(\%)\end{array}$ & $\begin{array}{l}\text { Peripheral } \\
(n=310) \\
(\%)\end{array}$ & $\begin{array}{l}\text { Control } \\
(n=504) \\
(\%)\end{array}$ \\
\hline A & 62.7 & 44.2 & 89.7 \\
B & 1.9 & 3.2 & 6.5 \\
C & 8.1 & 15.5 & 1.0 \\
D & 6.5 & 13.6 & 1.0 \\
E1 & 12.3 & 12.3 & 1.0 \\
E2 & 8.5 & 11.2 & 0.8 \\
\hline
\end{tabular}

${ }^{\mathrm{a}} n=$ Number of metaphase plates evaluated

Mitotic cells, 260 and 310, respectively, were obtained 7 to $9 \mathrm{~h}$ after central and peripheral microirradiation and caffeine posttreatment $(0.5 \mathrm{mM})$. Mitotic cells were classified into classes A-E as previously described (Cremer et al. 1981), (Table 1). For short definitions see legend of Table 1. Note that similar percentages of cells with GCS (class E) were found after central and peripheral microirradiation ( $20.8 \%$ vs $23.5 \%$ ). The percentages of classes $\mathrm{C}$ (PCS) and D were somewhat higher after peripheral as compared to central microirradiation. While the reason of this difference is not clear, it is important to note that both PCS and GCS can be induced by microirradiation of any site of the nucleus. In general, the probability of hitting targets important for the induction of chromosome shattering appears to be similar at different sites of the nucleoplasm.

In some of the experiments ${ }^{3} \mathrm{H}$-thymidine $(0.05 \mu \mathrm{Ci} / \mathrm{ml})$ was added to the cells during the caffeine postincubation period. In these experiments 461 mitotic cells were obtained after central and peripheral microirradiation, respectively. A similar number of non-irradiated mitotic cells in the same dishes were evaluated as controls. The percentage of labelled non-irradiated cells $(91.8 \%)$ did not significantly differ from the one obtained for labelled microirradiated cells $(93.9 \%)$. Cells with GCS and generally also with PCS (Fig. 1 a,b) showed label distributed over the whole chromatin. This indicates that these cells had traversed a considerable part of S-phase after microirradiation. In some cases of PCS, however, label was mainly or even completely restricted to the $\mathrm{X}$-chromosomes, while the area of shattered chromatin appeared free from label (Fig. 1 c,d). In Chinese hamster cells it has been shown that both the active and inactive $\mathrm{X}$-chromosome contain large amounts of late replicating chromatin (Daeven and Petersen 1973). Notably, ${ }^{3} \mathrm{H}$-thymidine was only added after microirradiation of all cells in the experimental field of a petri dish was finished. This means a maximum time delay of $1 \mathrm{~h}$ between microirradiation of a particular cell and addition of the label. Since replication of the
UV-irradiated chromatin appears necessary in order to induce shattering by the synergistic effect of caffeine (Nilsson and Lehmann 1975; Cremer et al. 1981), lack of label over the area of shattered chromosomes is considered to indicate that DNA replication critical for the induction of shattering was finished in the microirradiated chromatin during this time delay.

After Q-banding, karyotypes could be established from 21 mitotic cells with PCS after central microirradiation and from 29 mitotic cells after peripheral microirradiation. In some cases the structure and banding pattern of damaged chromosomes was still sufficiently maintained to allow direct identification. In other cases, damaged chromosomes could only be assessed in an indirect way after the identifiable intact or less damaged chromosomes had been arranged in the karyotypes. The results are shown in Tables 2 and 3 . The number of damaged chromosomes per spread $(2 n=22)$ varied from $1-11$. On average. 5.9 chromosomes were damaged after central microirradiation and 5.0 after peripheral microirradiation. Tables 2 and 3 show that each chromosome of the complement could be damaged by both central and peripheral microirradiation. When the binomial assumption was made, differences obtained for the relative frequencies of damage at either site of microirradiation (Fig. 2) were not significant at the $95 \%$ confidence level.

A significant increase $(P<0.05)$ of the frequency of damage was noted with increasing DNA content of chromosomes (Gray et al. 1975) by linear regression analysis with a regression coefficient $a=+0.26$ (Fig. 2, see slope of regression line) and a correlation coefficient $r=0.65$. The same correlation $(a=+0.25$; $r=0.64$ ) was observed, when the relative length of metaphase chromosomes from 80 metaphase spreads was determined. For this purpose the length of chromosome No. 1 was arbitrarily set at 1.0 (data not shown).

The type of damage which we should expect to result from microirradiation of somatically paired homologous chromosomes is exemplified in Fig. 3. Here, microirradiation was by chance performed in a cell with endoreduplication. In the resulting metaphase spread diplochromosomes appear either both intact or damaged at corresponding sites. As shown below, such a close and regular association, however, does not hold true for homologous chromosomes. For each pair of chromosome we have determined from Tables 2 and 3 the total number of metaphase spreads with PCS showing damage of either one or both chromosomes of the pair in question, the fraction of spreads showing damage to both homologous simultaneously and the fraction of spreads showing damage to only one chromosome (Table 4). With one exception simultaneous damage of homologous chromosomes was obtained in a minor fraction of the spreads. Only after central microirradiation did both X-chromosomes show simultaneous damage in the majority of cases (seven spreads showing damage of both $\mathrm{X}$-chromosomes versus five spreads showing damage of one $\mathrm{X}$ only).

Distribution of Homologous Chromosomes in Metaphase Spreads from Microirradiated and Non-Irradiated Cells. Centromerecentromere distances between homologous chromosomes were measured in 210 non-irradiated cells and in 50 cells with PCS. $\Delta^{2}$-distribution histograms of generalized centromere-centromere distances for each pair of homologous chromosomes were compared by $\chi^{2}$-tests with their respective reference distribution. This reference distribution was established from the generalized distances obtained for all possible combinations of homologous and non-homologous chromosome pairs except for the pair under study. For comparison a modified reference distribution 
Table 2. Partial chromosome shattering in microirradiated cells. I. Central microirradiation. Q-banded metaphase spreads with PCS (Roman numerals, vertical row) were obtained after central microirradiation karyotyped according to Kato and Yosida (1972) with the X-chromosome (No. 3) placed in sequence with the autosomes in descending size (arabic numerals, horizontal row). The smallest chromosomes, No.9-11, were grouped together. For each chromosome of a spread, damage is indicated by "*". Joint damage of homologous chromosomes is indicated by "**" for chromosomes 1-8. The number of damaged chromosomes $(n)$ in each spread is given on the right. At the bottom of each vertical row the total number $(N)$ is given, with which an individual chromosome has participated in PCS. $N(\%)$ shows the relative frequency of damage as percent of the theoretical limit of $N$, which would be reached, when a pair of homologous chromosomes would participate in PCS in all evaluated metaphase spreads. $N(\%)=$ (number of damaged chromosomes of a given type/number of all chromosomes of this type in the evaluated metaphase spreads) $\times 100$

\begin{tabular}{|c|c|c|c|c|c|c|c|c|c|c|}
\hline & \multicolumn{9}{|c|}{ Chromosome number } & $\begin{array}{l}\text { Number of } \\
\text { damaged } \\
\text { chromosomes } \\
(n)\end{array}$ \\
\hline I & & * & $*$ & * & * & $*$ & $*$ & $*$ & * & 8 \\
\hline II & & & & & * & * & * & $*$ & * & 5 \\
\hline III & & $*$ & & $*$ & $*$ & & & & $*$ & 4 \\
\hline $\mathrm{V}$ & & $*$ & & & & & & & & 1 \\
\hline VI & & $*$ & $* *$ & & * & * & & $* *$ & * & 8 \\
\hline VII & $*$ & $*$ & $* *$ & & * & $*$ & $*$ & $*$ & $*$ & 9 \\
\hline VIII & $*$ & $*$ & $*$ & & $*$ & $*$ & & & & 5 \\
\hline XIII & * & & & & & & & * & * & 3 \\
\hline $\mathrm{XIV}$ & $*$ & $*$ & * & $* *$ & & & $*$ & & $*: * *$ & 9 \\
\hline $\mathrm{XV}$ & $*$ & & & $*$ & * & & & & & 3 \\
\hline XVI & $*$ & & $* *$ & & $* *$ & $* *$ & $*$ & & $*$ & 9 \\
\hline XVII & $* *$ & $*$ & $* *$ & $* *$ & & & $*$ & & $* * *$ & 11 \\
\hline XVIII & $* *$ & & & & $*$ & & & & $*$ & 4 \\
\hline XIX & $*$ & $*$ & $* *$ & & $*$ & & & & & 5 \\
\hline$X X$ & $*$ & & $*$ & $*$ & $* *$ & $* *$ & $* *$ & $*$ & & 10 \\
\hline XXI & $*$ & & $*$ & $*$ & & $*$ & $*$ & & $* * *$ & 8 \\
\hline
\end{tabular}

was also used, namely the histogram established from generalized distances between the centromeres of the chromosome pair under study and the centromeres of all other non-homologous chromosomes. In addition to the centromere-centromere distance, the angle between the centromere of a chromosome under study, the centre of the metaphase spread and the centromere of the homologous chromosome was measured in the nonirradiated cell population. The resulting histogram was compared by $\chi^{2}$-test with the histogram established from the angles for all other possible pairs of chromosomes as reference distribution. In both the microirradiated and the non-irradiated cell population, evaluation of generalized distances between chromosomes as well as angle analysis consistently showed that homologous metaphase chromosomes were not situated closer to each other than expected by the reference distribution (data not shown).

Centromere-center Distances in Metaphase Spreads from Microirradiated and Non-Irradiated Cells. The same populations of metaphases with PCS and control metaphases were also evaluated for centromere-center distances. For each pair of homologous chromosomes the $\mathrm{d}^{2}$-distribution histogram of generalized centromere-center distances was compared by $\chi^{2}$-tests with the respective reference distribution. i.e., the histogram established by the $\mathrm{d}^{2}$-values of all chromosome pairs except the one under consideration. For most chromosome pairs $\chi^{2}$-tests did not reveal a significant deviation from the reference distribution (data not shown). In the non-irradiated cell population the centromeres of chromosomes No. 1 appeared more central $(P<0.05)$, while the centromeres of chromosomes No. 6 and 11 were preferentially located at the periphery of the spreads $(P<0.05$ and $P<0.025$ respectively). In the sample of metaphases with PCS the centromeres of chromosome No.7 appeared more peripherally located $(P<0.05)$. The supposedly central or peripheral localization of these few chromosomes may represent statistical artifacts and further discussion would only be worthwhile if the same localization could be consistently found in several independent series of experiments. No signifi- 
Table 3. Partial chromosome shattering in microirradiated cells. II. Peripheral microirradiation. Q-banded metaphase spreads (Roman numerals, vertical row) were obtained after peripheral microirradiation and karyotyped according to Kato and Yosida (1972). For further details see legend of Table 2

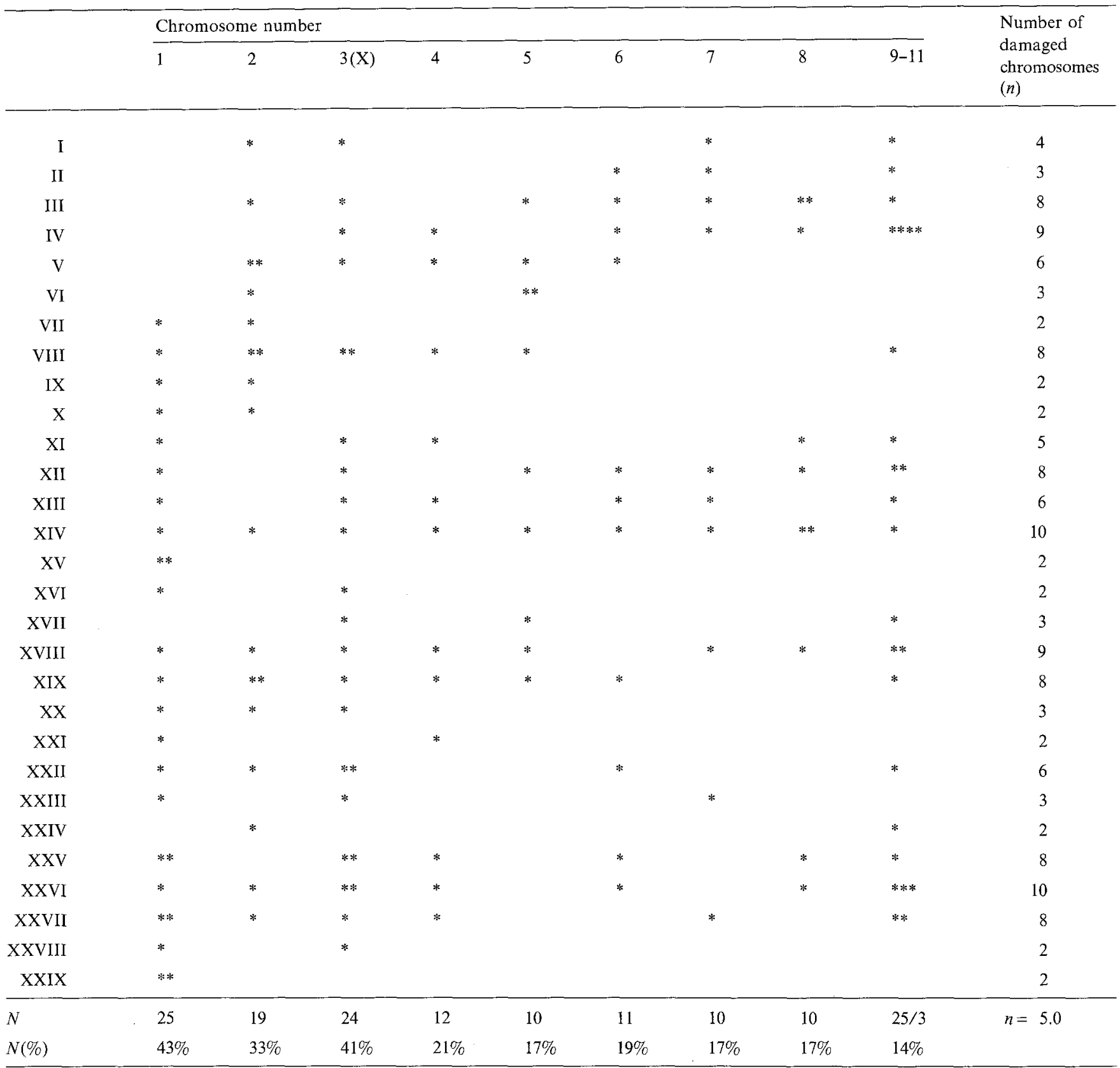

cant differences were observed when the centromere-centre distances of chromosomes in microirradiated cells, as measured for intact and damaged but still identifiable chromosomes, were compared by $\chi^{2}$-tests with the corresponding distances of chromosomes in non-irradiated cells (data not shown).

Distribution of Damaged Chromosomes in Metaphase Spreads with PCS. Metaphase spreads with PCS showed an area of fragmented or pulverized chromosome material. As noted above, damage in this area was normally too heavy to allow direct identification of chromosomes. While the majority of chromosomes surrounding the damaged area appeared intact, some of them also showed damage (breaks, miscondensation), but as a rule could still be identified. Table 5 shows that these less damaged chromosomes were situated significantly closer to the damaged area than intact ones. For this evaluation generalized distances $\left(\Delta^{2}\right)$ of the centromeres of any damaged chromosome to the arbitrarily defined center of the damaged area (DA) were compared with $\bar{\Delta}^{2}$-distances of any intact chromosome to this center.

\section{Discussion}

This study deals with the evaluation of metaphase spreads displaying shattered chromosomes after microirradiation of the interphase nucleus of CHL-cells either at its central area or at its edge. It contributes to the analysis of both the mechanism of chromosome shattering by the synergistic effect of ultraviolet light and caffeine (for review see Kihlman 1977) and the 


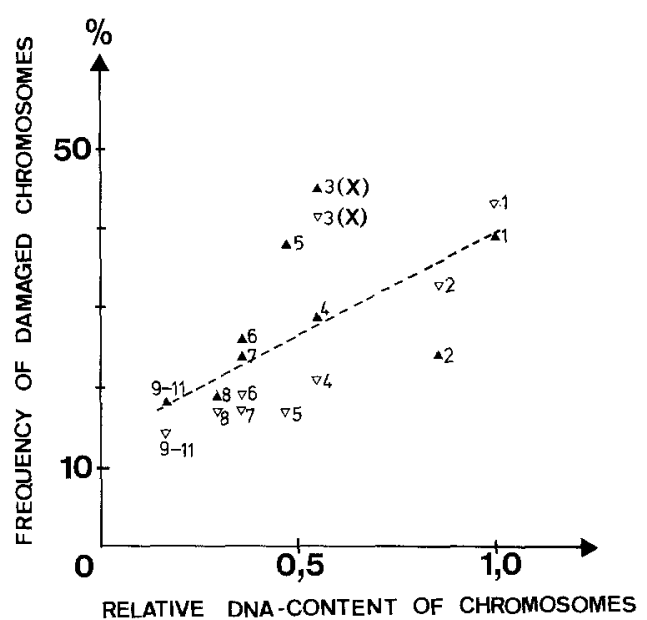

Fig. 2. Abscissa. The relative DNA-content of Chinese hamster chromosomes was derived from the literature (Gray et al. 1975). The DNA content of the largest chromosome (No.1) was arbitrarily set at 1.0. Ordinate. The relative frequency $[N(\%)$; see legend to Table 2] with which a particular chromosome participated in PCS either after peripheral $(\nabla)$ or after central ( $\Delta$ ) microirradiation, was calculated from data shown in Tables 2 and 3. The dotted line represents the linear regression line calculated from all points of the plot. Note that the regression coefficient as indicated by the slope of this line is positive $(+0.26)$. This increase in the frequency of damage with the DNA content of single chromosomes is significant $(P<0.05)$

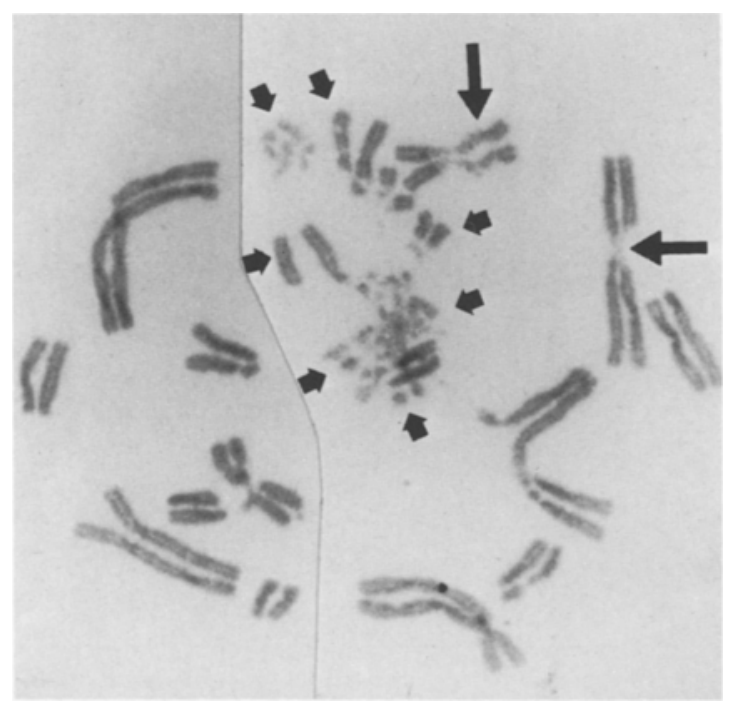

Fig. 3. This Chinese hamster cell (V79-subline; Cremer et al. 1976, 1981a, b) was microirradiated at one site of the nucleoplasm and post-treated with $1 \mathrm{mM}$ caffeine for $8 \mathrm{~h}$ before chromosome preparation in situ was performed. Colchicine $(1 \mu \mathrm{g} / \mathrm{ml})$ was added $5 \mathrm{~h}$ after microirradiation. By chance endoreduplication had occurred in this cell. Small arrows indicate a damage area consisting of shattered diplochromosomes. Large arrows indicate diplochromosomes which are jointly affected at corresponding sites. The distribution of chromosomal damage in this metaphase spread is consistent with the assumption that each diplochromosome forms its own territory in the interphase nucleus

interphase arrangement of chromosomes. The results are compared with data obtained by the analysis of chromosome distribution in metaphase spreads.

The phenomena of partial (PCS) and generalized (GCS) chromosome shattering in microirradiated nuclei have been described extensively (Cremer et al. $1981 \mathrm{a}, \mathrm{b}$; Cremer et al. 1983). For a relevant discussion of presently conceivable
Table 4. Frequency of damage to chromosomes 1-8 as determined from Tables 2,3

\begin{tabular}{lllll}
\hline $\begin{array}{l}\text { Site of } \\
\text { micro- } \\
\text { irradiation }\end{array}$ & $\begin{array}{l}\text { Chromo- } \\
\text { some } \\
\text { no. }\end{array}$ & $\begin{array}{l}\text { Number } \\
\text { of meta- } \\
\text { phases }\end{array}$ & $\begin{array}{l}\text { One } \\
\text { chromo- } \\
\text { some } \\
\text { damaged }\end{array}$ & $\begin{array}{l}\text { both } \\
\text { chromosomes } \\
\text { damaged }^{\mathrm{c}}\end{array}$ \\
\hline Central & 1 & 14 & 0.86 & 0.14 \\
& 2 & 10 & 1.00 & 0.00 \\
& 3 & 12 & 0.42 & 0.58 \\
& 4 & 10 & 0.80 & 0.20 \\
& 5 & 13 & 0.77 & 0.23 \\
& 6 & 9 & 0.78 & 0.22 \\
& 7 & 9 & 0.89 & 0.11 \\
\hline Peripheral & 8 & 7 & 0.86 & 0.14 \\
& 1 & 21 & 0.81 & 0.19 \\
& 2 & 16 & 0.82 & 0.18 \\
& 3 & 20 & 0.80 & 0.20 \\
& 4 & 12 & 1.00 & 0.00 \\
& 5 & 9 & 0.89 & 0.11 \\
& 6 & 11 & 1.00 & 0.00 \\
& 7 & 10 & 1.00 & 0.00 \\
& 8 & 8 & 0.75 & 0.25 \\
\hline
\end{tabular}

${ }^{a}$ Chromosomes No.9-11 were grouped together (see Tables 2, 3); therefore these cases were omitted from Table 4.

b (Number of cases with damage to one chromosome of a given type)/ (total number of metaphases with damage to chromosomes of a given type)

c (Number of cases with damage to both homologous chromosomes of a given type)/(total number of metaphases with damage to chromosomes of a given type)

Table 5. Spatial distribution of chromosomes in cells with PCS. $\bar{\Delta}^{2}$ distances of any damaged chromosome to the damaged area (DA) compared with $\triangle^{2}$ distances of any undamaged chromosome to the damaged area (DA)

\begin{tabular}{|c|c|c|c|c|}
\hline & \multirow{3}{*}{$\begin{array}{l}\bar{\Delta}^{2} \text {-value } \\
\text { (DA-chro- } \\
\text { mosome) }\end{array}$} & \multicolumn{3}{|c|}{$\chi^{2}$ Comparison } \\
\hline & & \multicolumn{3}{|c|}{$\begin{array}{l}\text { Damaged versus } \\
\text { undamaged chromosomes }\end{array}$} \\
\hline & & $\chi^{2}$-value & $d f$ & $P$ \\
\hline $\begin{array}{l}\text { Damaged } \\
\text { chromosomes }\end{array}$ & 3.02 & 53.22 & 7 & $P<0.005$ \\
\hline $\begin{array}{l}\text { Undamaged } \\
\text { chromosomes }\end{array}$ & 4.28 & & & \\
\hline
\end{tabular}

$d f=$ degrees of freedom

mechanisms of GCS see Cremer et al. (1981a,b). The following discussion is restricted to the PCS-phenomenon and its relevance to the analysis of the interphase arrangement of chromosomes.

Since it is well established that DNA replication is delayed by the presence of pyrimidine dimers (Sauerbier 1976), it has been suggested that the cell might enter mitosis before replication is completed in the microirradiated nuclear segment (Zorn et al. 1976). PCS then might be due to premature condensation of still replicating microirradiated chromosome segments. In this case, one should expect incorporation of a late pulse of ${ }^{3} \mathrm{H}$-thymidine into the shattered chromatin but not into intact chromosomes. Present data are contrary to such a view. Cases have been 
observed in which the late replicating $\mathrm{X}$-chromosomes appear intact and have incorporated ${ }^{3} \mathrm{H}$-thymidine at a time when the shattered chromosomal segments had already finished DNAreplication.

Several lines of evidence support the hypothesis that chromosomal damage in cells with PCS is restricted, at least to a large extent, to the microirradiated chromosomes. Our data show that any chromosome of the complement could participate in PCS and that damaged chromosomes in general were clustered around a damage center.

The strongest piece of evidence stems from indirect immunofluorescence studies with antibodies against UV-irradiated DNA (Cremer et al. 1983, see Introduction). Interestingly, even in the case of PCS shattering often included somewhat more chromosome material than could be specifically stained. The occurrence of shattered but non-labelled chromatin clustered around the labelled region can possibly be attributed to stray light, which might produce a small amount of DNA-photolesions in the nuclear area close to the focal site of the microbeam. The number of these lesions might still be sufficient to induce shattering but not sufficient to be detected by indirect immunofluorescence microscopy. This interpretation would also explain why the amount of chromatin involved in PCS is often larger than one would expect from the focal beam diameter $(1-2 \mu \mathrm{m})$ even if one takes into account the aperture angle of the microbeam. While the following interpretation of PCS-data in terms of an interphase chromosome arrangement appears clearly justified by the above arguments, some restrictions should be taken into consideration. Classes D and E (GCS) show that chromosomes remote from the microirradiated nuclear segment can participate in chromosome shattering under certain conditions. To a limited extent such an indirect effect of microirradiation may also account for chromosomal damage in case of PCS. For example, the remarkable frequency of shattered $\mathrm{X}$-chromosomes in this series of experiments may reflect such a phenomenon and not only be due to the frequency with which these chromosomes were hit by the microbeam. Previously, we have shown that the frequency of PCS increases when microirradiation is performed at later S-phase as compared to G1/early S-phase (Cremer et al. 1981 a). Since DNA replication following microirradiation is a necessary event for the induction of chromosome shattering, one may expect an increased sensitivity of late replicating as compared to early replicating chromatin. This line of argument suggests that the frequency with which a particular chromosome participated in PCS may not only depend on the frequency with which it was situated in the microirradiated nuclear area but also on the other factors such as differences between the individual replication patterns of chromosomes. The present investigation has shown that each chromosome can participate in PCS with a frequency roughly corresponding to its DNA content. This finding would easily be explained by the assumption that the likelihood of each chromosome being hit by the microbeam depends mainly on the size of its interphase territory and that this size is related to its DNA content, although other parameters, for example genetic activity/inactivity of chromatin, are likely to influence the size of chromosome territories as well.

The time protocol of the present experiments suggests that the majority of the cells with PCS were in S-phase at the time of microirradiation. For a number of cells this was confirmed by labelling studies with ${ }^{3} \mathrm{H}$-thymidine. PCS resulting from microirradiation of S-phase nuclei gives further support to the idea that the territorial organization of the interphase nucleus as described for cells in G1 (Zorn et al. 1979; Cremer et al. 1982 ) is maintained in S-phase. Our view that chromosomes increase their volume when they pass from mitosis to interphase but still form distinct domains has already been proposed by Rabl (1885) and Boveri (1909). Such a view predicts certain limitations to the way in which chromatin fibres of individual chromosomes can be ordered in the interphase nucleus. While a territorial organization of interphase chromosomes appears to be most convenient for the interphasemetaphase transition of chromosome structure, such an organization by no means follows directly from the now firmly established paradigm of chromosome individuality. It is interesting to note that Boveri (1909) was well aware that this paradigm would be compatible with very different types of chromatin distribution. Since then direct evidence for a territorial organization of interphase chromosomes has remained meagre (for review see Comings 1980) and not long ago arrangements of chromatin fibres were discussed which are contrary to such a view (Comings 1968; Vogel and Schroeder 1974). Thus the concept of chromosome territories so far has been accepted by many scientists rather for its convenience than because of the necessity for experimental proof. Our microbeam approach provides the first method, by which the interphase distribution of euchromatic or heterochromatic parts of any individual chromosome can be directly visualized.

Frequencies of damage obtained for individual chromosomes did not significantly differ after central and peripheral microirradiation, respectively. Damage of a chromosome at either site of microirradiation can be explained in two ways.

(i) Chromosome territories extend from the periphery to the central part of the nucleus or even to the opposite site of the nuclear edge. Previously, we have presented evidence for a polarization of chromosomes in CHL-interphase nuclei as a passive relic of their anaphase-telophase orientation (Cremer et al. 1982). If chromosome territories were arranged like pieces of a pie with the centromeres co-orientated to each other in the central nuclear part we would expect the number of damaged chromosomes to be higher after central microirradiation. However, the average number of chromosomes damaged by central or peripheral microirradiation was rather similar (5.9 versus 5.0).

(ii) The central or peripheral position of whole territories varies in different interphase nuclei. Our data argue against the idea that certain chromosome territories as a whole maintain fixed positions in the central part of the CHL-nucleus, while others exclusively contribute to its periphery. Variation in the position of chromosomes in different interphase nuclei is also indicated by varying combinations of shattered chromosomes both after central and peripheral microirradiation. Evaluation of a sufficient number of metaphase spreads with PCS should make it possible to detect non-random arrangements, if any of them are present in the nucleus of CHL-cells. The question of nonrandom associations between territories of non-homologous chromosomes must be shelved until more data become available. Some discussion of the question of whether a non-random association of homologous chromosomes exists in CHL-interphase nuclei, however, seems worthwhile at the present stage.

Joint damage of homologous chromosomes was observed in a minority of cases, although on average 5-6 chromosomes were damaged. The fractions of spreads showing simultaneous damage of homologous chromosomes have to be compared with the fractions obtained for the simultaneous damage of the chromosome under consideration with other non-homologous chromosomes. In case of random association of the territories of 
homologous and non-homologous chromosomes we should expect that simultaneous damage of the chromosome under consideration and of a non-homologous chromosome of similar size occurs approximately twice as often as with its homologous counterpart. The reason for this is simply that there are two nonhomologous chromosomes but only one homologous counterpart. The frequencies of joint damage between the possible combinations of non-homologous chromosomes are not shown, but can easily be obtained from Tables 1 and 2 . When examining these data in the light of the above considerations we do not note an obvious excess of simultaneous damage of homologous chromosomes over random expectation.

As noted above the frequency with which $\mathrm{X}$-chromosomes were jointly damaged might be influenced by other factors than their neighbourship in the interphase nucleus. In contrast, joint damage of diplochromosomes was observed when an endoreduplicating nucleus was microirradiated by chance. While our data clearly argue against somatic pairing of homologous chromosomes as a regular event, occasional somatic pairing or pairing restricted to certain segments of homologous chromosomes is still possible. Indeed, evidence for mitotic crossing over taking place in human cells has been presented by Therman and Meyer-Kuhn (1981). The final answer to the distribution of homologous chromosomes in the interphase nucleus of mammalian cells has to be based on more data and more statistical refinement.

The last part of this discussion is devoted to the relationship between the position of chromosome territories in the interphase nucleus and the position of chromosomes in the metaphase spread. The validity of the many analyses of metaphase chromosome arrangements for obtaining information on the chromosome arrangement in interphase has been severely doubted for a number of reasons. A priori, such an approach seems only worthwhile in case of a territorial organization of interphase chromosomes which retain their relative positions throughout interphase. Many authors have simply presumed these conditions without presenting particular evidence for them. While our microirradiation experiments strongly support these assumptions for the cell type studied by us (Cremer et al. 1982), other important objections have now to be considered. Firstly, the two-dimensional distribution of chromosomes in the metaphase spread is derived from a three-dimensional one in the interphase nucleus. This problem may be more severe in a spherical nucleus (e.g. in lymphocytes) than in a rather flat one (e.g. in CHL-cells). Secondly, many analyses are based on the metaphase distribution of centromeric regions, i.e. centromerecentromere and centromere-center analyses. Additional information concerning the distribution of interphasic centromeres and the extent of their possible redistribution between late $G 2$ and metaphase is paramount when one tries to interprete such data in terms of an interphase chromosome arrangement. For example, it makes an important difference whether centromeres are clustered in a limited portion of the nucleus either at the nuclear edge (Rabl 1885) or elsewhere (Del Fosse and Church 1981) or distributed largely at random (Brenner et al. 1981). Recent studies of the distribution of interphasic centromeres indicate considerable variation in cell types from different plant and mammalian species (Church and Moens 1976; Del Fosse and Church 1981; Moroi et al. 1981; Brenner et al. 1981). Thirdly, and probably most important, the technical procedures routinely used for obtaining well spread metaphases (often including colchicine treatment and hypotonic shock) alter the chromosome arrangement of the intact metaphase plate to an extent which has not been documented by most investigators, although it can be shown that the relationship between chromosome arrangements at interphase and metaphase is critically influenced by the experimental regimen followed (Schmid et al. 1981).

Experimentally, such a relationship can be tested in two ways. Firstly, the results of statistical analysis of chromosome arrangements at metaphase are compared with the results of chromosome arrangements at interphase obtained with the microbeam or other independent methods (Schmid et al. 1981; Dutrillaux et al. 1981; Hager et al. 1982; Hens et al. 1982). The variability of interphase chromosome arrangements as indicated by the present microbeam study is in agreement with the variability of chromosome positions and the lack of association between homologous chromosomes in the metaphase spread. Unfortunately, our present results do not bear on the question of the validity of the latter type of analysis. In this respect, correspondence of data is only meaningful if non-random arrangements can be shown to either persist or become lost when the cell proceeds from interphase to metaphase.

Secondly microirradiation itself provides a means to mark a few chromosome territories at interphase and to follow their relative positions up to metaphase. As we have already mentioned, it can be shown that the population of damaged chromosomes is not randomly distributed in metaphase spreads with PCS but clustered around a damage center. It should be noted, however, that damaged chromosomes might show enhanced "stickiness" towards each other and thus might not be representative for the interphase-metaphase relationship of intact chromosomes. Further studies are now under way to fully exploit this approach. In one study sister chromatid exchanges are induced in microirradiated chromosome territories and the distribution of chromosomes with high levels of SCEs is tested in the subsequent metaphase (Raith et al. in preparation). In another study microirradiated chromatin is followed by indirect immunofluorescence through the subsequent interphase and mitosis (Hens et al. in preparation).

Acknowledgements. This work has been supported by the Deutsche Forschungsgemeinschaft. We thank Professor T. M. Schroeder, Professor F. Vogel, and Professor U. Wolf for their continuous interest in this study and J. Krüger for statistical advice. The technical assistance of Miss Nadja Bulbuc is gratefully acknowledged.

\section{References}

Avivi L, Feldman M (1980) Arrangement of chromosomes in the interphase nucleus of plants. Hum Genet 55:281-295

Barton DE, David FN (1962) The analysis of chromosome patterns in the normal cell. Ann Hum Genet 25:323-329

Boveri Th (1909) Die Blastomerenkerne von Ascaris megalocephala und die Theorie der Chromosomenindividualität. Arch Zellforsch 3: $181-268$

Brenner S, Pepper D, Berns MW, Tan E, Brinkley BR (1981) Kinetochore structure, duplication, and distribution in mammalian cells: analysis by human autoantibodies from scleroderma patients. J Cell Biol 91:95-102

Caspersson T, Zech L, Johansson C (1970) Differential binding of alkylating fluorochromes in human chromosomes. Exp Cell Res 60: 315-319

Church K, Moens PB (1976) Centromere behavior during interphase and meiotic prophase in Allium fistulosum from 3-D, E.M, reconstruction, Chromosoma 56:249-263

Comings DE (1968) The rationale for an ordered arrangement of chromatin in the interphase nucleus. Am J Hum Genet 20:440-460

Comings DE (1980) Arrangement of chromatin in the nucleus. Hum Genet 53:131-143 
Cremer C, Zorn C, Cremer T (1974) An ultraviolet microbeam for $257 \mathrm{~nm}$. Microsc Acta. 75:331-337

Cremer C, Cremer T, Zorn C, Schoeller L (1976) Effects of laser-UVmicroirradiation $(\lambda=2573 \AA)$ on proliferation of Chinese hamster cells. Radiat Res 66:106-121

Cremer C, Cremer T, Fukuda M, Nakanishi K (1980) Detection of laserUV-microirradiation induced DNA photolesions by immunofluorescent staining. Hum Genet 54:107-110

Cremer C, Cremer T, Zorn C, Zimmer J (1981 a) Induction of chromosome shattering by ultraviolet light and caffeine: comparison of whole-cell and partial-cell irradiation. Mutat Res $84: 331-348$

Cremer T, Peterson SP, Cremer C, Berns MW (1981b) Laser microirradiation of Chinese hamster cells at wavelength $365 \mathrm{~nm}$ : effects of psoralen and caffeine. Radiat Res $85: 529-543$

Cremer T, Cremer C, Baumann H, Luedtke E-K, Sperling K, Teuber V, Zorn C (1982) Rabl's model of the interphase chromosome arrangement tested in Chinese hamster cells by premature chromosome condensation and laser-UV-microbeam experiments. Hum Genet $60: 46-56$

Cremer C, Cremer T, Hens L, Baumann H, Cornelis JJ, Nakanirhi K (1983) UV-microirradiation of the Chinese hamster cell nucleus and caffeine posttreatment: immunocytochemical localizations of DNA photolesions in cells with partial and generalized chromosome shattering. Mutat Res, in press

Deaven LL, Petersen DF: (1973) The chromosomes of CHO, an aneuploid Chinese hamster cell line: G-band, C-band, and autoradiographic analyses. Chromosoma $41: 129-144$

Del Fosse FE, Church K (1981) Presynaptic chromosome behavior in Lilium. I. Centromere orientation and movement during premeiotic interphase in Lilium speciosum cv. Rosemede. Chromosoma 81: $701-716$

Dutrillaux B, Viegas-Péquignot E, Aurias A, Mouthuy M, Prieur M (1981) Non-random position of metaphasic chromosomes: a study of radiation induced and constitutional chromosome reartangements. Hum Genet $59: 208-210$

Gray JW, Carrano AV, Steinmetz CC, van Dilla MA, Moore II DH, Mayall BH, Mendelsohn CM (1975) Chromosome measurement and sorting by flow systems. Proc Natl Acad Sci USA 72:1231-1234

Hager HD, Schroeder-Kurth TM, Vogel F (1982) Position of chromosomes in the human interphase nucleus. An analysis of nonhomologous chromatid translocations in lymphocyte cultures after Trenimon treatment and from patients with Fanconi's anemia and Bloom's syndrome. Hum Genet 61:342-356
Hens L (1976) Chromosome distribution in $\mathrm{a}_{23}$ Chinese hamster fibroblasts. Chromosoma 57:205-217

Hens L, Kirsch-Volders M, Verschaeve L, Susanne C (1982) The central localization of the small and early replicating chromosomes in human diploid metaphase figures. Hum Genet, 60:249-256

Kato H, Yosida TH (1972) Banding patterns of Chinese hamster chromosomes revealed by new techniques. Chromosoma 36: 272280

Kihlman BA (1977) Caffeine and chromosomes. Elsevier, Amsterdam

Moroi Y, Hartman AL, Nakane PK, Tan EM (1981) Distribution of kinetochore (centromere) antigen in mammalian cell nuclei. J Cell Biol 90:254-259

Nilsson K, Lehmann AR (1975) The effect of methylated oxypurines on the size of newly-synthesized DNA and on the production of chromosome aberrations after UV-irradiation in Chinese hamster cells. Mutat Res 30:255-266

Rabl C (1885) Ưber Zelltheilung. In: Gegenbaur C(ed) Morphologisches Jahrbuch 10:214-330

Ray M, Mohandas T (1976) Proposed banding nomenclature for the Chinese hamster chromosomes. Cytogenet Cell Genet 16:83-91

Sauerbier W (1976) UV-damage at the transcriptional level. Adv Radiat Biol 6:161-210

Schmid M, Poppen A, Schmid W, Engel W (1981) Somatic pairings of the Y heterochromatin in human XYY and XYqi cells. Cytogenet Cell Genet 29:203-214

Therman E, Meyer Kuhn E (1981) Mitotic crossing-over and segregation in man. Hum Genet 59:93-100

Vogel F, Schroeder TM (1974) The internal order of the interphase nucleus. Hum Genet 25:265-297

Wollenberg C, Kiefaber MP, Zang KD (1982) Quantitative studies on the arrangement of human metaphase chromosomes. VIII. Localization of homologous chromosomes. Hum Genet, 60:239-248

Zorn C, Cremer T, Cremer C, Zimmer J (1976) Laser-UV-microirradiation of interphase nuclei and posttreatment with caffeine: a new approach to establish the arrangement of interphase chromosomes. Hum Genet 35:83-89

Zorn C, Cremer C, Cremer T, Zimmer J (1979) Unscheduled DNA synthesis after partial UV-irradiation of the cell nucleus. Distribution in interphase and metaphase. Exp Cell Res 124:111-119

Received September 1, 1982 